\title{
A Method for Improving the Accuracy of Natural Frequency Measurement Using In-the-loop Computing
}

\author{
Adam Kotowski \\ Faculty of Electrical Engineering, Bialystok University of Technology, Wiejska, 45D, 15-351 Bialystok, Poland, \\ a.kotowski@pb.edu.pl
}

\begin{abstract}
The method presented in the paper is based on in-the-loop computing applied for impulse response to obtain a spectrum with a much higher frequency resolution than using FFT. Then, higher spectrum frequency resolution results in greater accuracy in estimation of natural frequencies. The frequency resolution of estimated spectrum in this method is completely independent of the length of impulse response and, by extension, the method eliminates the problem of spectral resolution limitation using FFT due to finite length of recorded signals. This fact is very useful and is the main advantage of the proposed method. The results of the method have been shown and compared in quantitative terms to natural frequencies estimated using classical FFT with zero-padding as reference method.
\end{abstract}

Keywords: Natural frequency, measurement accuracy improvement, in-the-loop computing, impulse response.

\section{INTRODUCTION}

In many technical applications it is very useful to measure vibration signals. These signals provide important information as the basis for damage detection of a single object or whole systems. Vibration-based damage detection techniques use many parameters obtained by signal processing methods [1], [2]. Some of these parameters can only be obtained when the machine is in motion. A good example is the use of vibration signals for diagnostic techniques of electrical motors described in [3]. The authors proposed a method of feature extraction for obtaining feature vectors. Then, the detection of deterioration of three-phase induction motor using vibration signals was possible to apply in practice. We have a significant example here that the deterioration of an object's state is successfully detected during its operation.

The situation is completely different when vibration signals are measured when the examined object is a single component of the machinery, disassembled and tested during the scheduled machine shutdown. In this case, we can use impulse tests. Having the impulse responses, we are able to estimate natural frequencies as fault-oriented parameters. The correctness of the damage detection depends on the accuracy of natural frequency measurement.

Natural frequency is one of the fundamental parameters in measurement of physical quantities of solids or systems. In addition, this indicator has its wide range of applications in the methods of damage detection in structures. Natural frequencies are sensitive to many kinds of damage and they are the most often used damage indicator both formerly and nowadays [4]-[8].

Vibration-based methods for natural frequency estimation have been widely studied, as they are non-destructive, inexpensive and convenient. Natural frequencies are estimated fast and in an easy way using only a single sensor trough impulse tests. Mode shapes require measurements at numerous locations so they need more time. Damping ratios are difficult to measure and sensitive to environmental factors such as variations in humidity and temperature [9]. Beside absolute values of natural frequencies, changes in natural frequencies may be also called the classical damage indicators. Changes in natural frequencies for detection of fatigue cracks have been often proposed, applied to many engineering structures [10], [11]. In addition, relative natural frequency changes are used in single and multiple damage detection [7]. The reduction in natural frequencies is an important information to detect cracks in structures. Therefore, an ability for accurate natural frequency estimation is very important in the field of fault diagnostics. Structural damage detection based on changes of natural frequencies has proved that both the changes in $\mathrm{Hz}$ and relative changes can be unnoticeable if frequency resolution of the spectrum is insufficient.

Differences in natural frequencies due to damage like cracks are at the level of several tenths of $\mathrm{Hz}$ or even at the level of several hundredths of $\mathrm{Hz}$ [7], [11]. Therefore, lots of algorithms have been proposed to provide accurate natural frequency estimation by improving frequency resolution of 
the spectra. Many interpolated FFT methods were proposed to obtain the accurate estimation [12]-[15] as well as Adaptive Notch Filter (ANF) [16]. The importance of accuracy in the process of measurements of the natural frequencies is presented in [17]. The precise natural frequency estimation algorithm was presented using the vibrational response of structure excited to cross through the resonance. Natural frequency estimates were achieved using swept-sine excitation.

In the case of spectral analysis based on FFT it is common knowledge that the frequency resolution $\Delta f_{F F T}$ is absolutely dependent on the length of the signal under analysis. Here, an error of natural frequency estimation is in the range $\pm \Delta \mathrm{f}_{\mathrm{FFT}} / 2$. Using the method proposed in the paper, the frequency resolution of the spectrum is completely independent of the signal length. Therefore, the frequency resolution and, hence, the accuracy of the natural frequency measurement can be significantly increased over the FFT.

\section{Methodology}

The method proposed in this Section is based on the multiplication of autocorrelation function of impulse response with harmonic wave. Using the proposed method it is possible to transform impulse response into its spectrum by arbitrary and freely adjustable frequency resolution. If frequency resolution for the spectra obtained using fast Fourier transform (FFT) is declared as $\Delta f_{F F T}=f_{s} / \mathrm{N}$, the new spectral resolution $\Delta f_{s c r}$ of the spectra obtained through the proposed method is independent of signal length $N$ and sampling frequency $f s$. The idea of the method consists of a loop as shown in the form of diagram in Fig.1. The algorithm presented here computes the values of lines of the discrete spectrum $S\left(f_{i}\right)$ against frequency $f_{i}$ with a step depending on the predetermined frequency resolution $\Delta f_{\text {scr. }}$.

At the very beginning, the method reads the recorded impulse response. Then, in the second step, the spectral resolution value $\Delta f_{s c r}$ is read. The resolution $\Delta f_{s c r}$ is user definable and can be integer or non-integer. The in-the-loop computation results in the spectrum of the impulse response at this frequency resolution. However, the spectral resolution $\Delta f_{s c r}$ may change during the operation of the proposed algorithm if needed. Therefore, the spectrum of impulse response can be computed by required frequency resolution only around the natural frequencies preliminary detected using FFT, e.g. one-tenth of $\Delta f_{F F T}$, one-hundredth of $\Delta f_{F F T}$. Thus, the accuracy of natural frequency measurement can be significantly improved and the amount of computation can be reduced.

Another preliminary operation of the algorithm is determining the auto-correlation function (ACF) of the impulse response. The ACF is defined as follows [18], [19]

$$
R_{x x}(\tau)=\lim _{T \rightarrow \infty} \frac{1}{T} \int_{0}^{T} x(t) x(t+\tau) d t
$$

where $x(t)$ represents impulse response and $\tau$ is delay time. The use of the ACF here will allow better observation of the dominant harmonic components existing in the impulse response.

After starting the algorithm in the loop, the cosine wave $x\left(f_{i}\right)$ is declared for discrete time vector $t_{k}\left(t_{k}=k \cdot \Delta t, \Delta t=1 / f_{s}\right.$, $k=0,1,2, . ., N-1)$. This wave is obtained as follows

$$
x\left(f_{i}\right)=\cos \left(2 \pi\left(i \cdot \Delta f_{s c r}\right) \cdot t_{k}\right)
$$

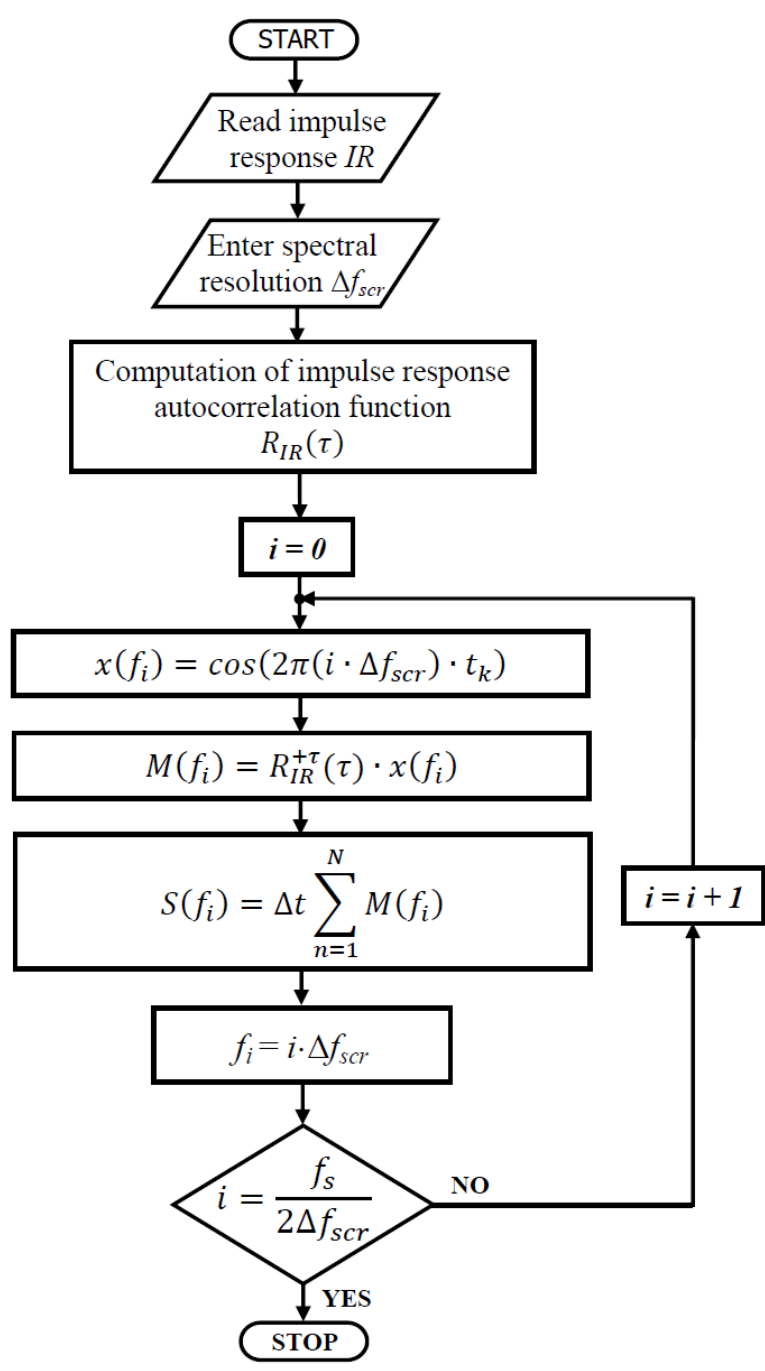

Fig.1. Diagram of the method for computing the spectrum with predefined frequency resolution.

In numerical calculations the length of data representing the autocorrelation function and cosine wave length should be equal. Therefore, subsequent calculations only take into account the ACF for positive time delay $\tau$ as follows

$$
R_{I R}^{+\tau}(\tau)=R_{I R}(\tau>0)
$$

The next calculations are executed in the loop for incrementing frequency of cosine wave until it reaches the upper limit of frequency range of the spectrum. This way, the frequency step constitutes frequency resolution of calculated 
spectrum and the method has resulted in the spectrum with own frequency resolution completely independent of impulse response length $N$.

The algorithm denotes values of multiplication of ACF and $x$ signal and it results in the $M\left(f_{i}\right)$ values. Next, spectrum $S\left(f_{i}\right)$ is obtained by summation of the $M\left(f_{i}\right)$ values and the sum obtained here is multiplied by sampling period $\Delta t$, where samples of input data vectors are numbered by $n=1 . . N$. Frequencies $f_{i}$ are growing linearly as the index $i$ increases from $i=0$ to $i=\left(f_{s} / 2\right) / \Delta f_{s c r}$, where $\Delta f_{s c r}$ means frequency resolution of the spectrum. Thus, the frequency $f_{i}$ is in the range from zero to the half of sampling frequency to fill up useful frequency range due to the Kotelnikov-Shannon theorem, up to the Nyquist frequency $f_{N y q}=f_{s} / 2$.

In general, the proposed method of spectrum calculation is based only on the autocorrelation function of analyzed impulse response and calculation of cosine wave to do simple mathematical functions.

\section{NUMERICAL STUDIES}

This chapter conducts a numerical experiment to apply the method described in Section 2. For this purpose, the impulse responses (IRs) of three degree-of-freedom (3-dof) system were analyzed. To illustrate the accuracy improvement of frequency determination trough spectral resolution improvement, a set of impulse responses of 3-dof system was generated. The natural frequencies have been estimated using in-the-loop method and classical FFT. It was made by finding the local maximum in the obtained spectrum.

The unit impulse response function of a multi degree-offreedom system can be expressed as [20]

$$
h(t)=\sum_{r=1}^{n} A_{r} e^{-\delta_{r} t} \sin \left(2 \pi \cdot f_{d r} \cdot t\right)
$$

where $A_{r}, \delta_{r}$, and $f_{d r}$ are the $r$-th modal constant, the $r$-th modal damping, and $r$-th damped natural frequency of the system, respectively.

To model impulse responses with randomized natural frequencies in this study, three ranges of natural frequency changes of 3-dof system were proposed. Thus, the generated impulse responses consist of harmonics with $f_{d 1}, f_{d 2}$, and $f_{d 3}$ frequencies in $(3-5) \mathrm{kHz},(12-15) \mathrm{kHz}$, and $(18-20) \mathrm{kHz}$ ranges, respectively. Exemplary synthesized impulse response is shown in Fig.2. and its spectra are shown in Fig.3.

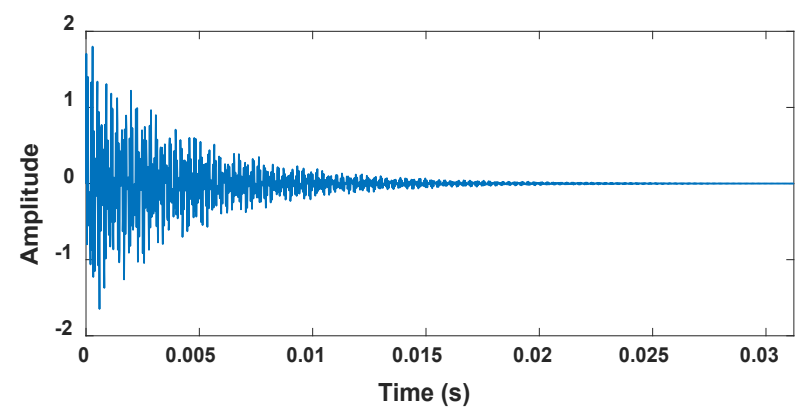

Fig.2. Impulse response for numerical studies.
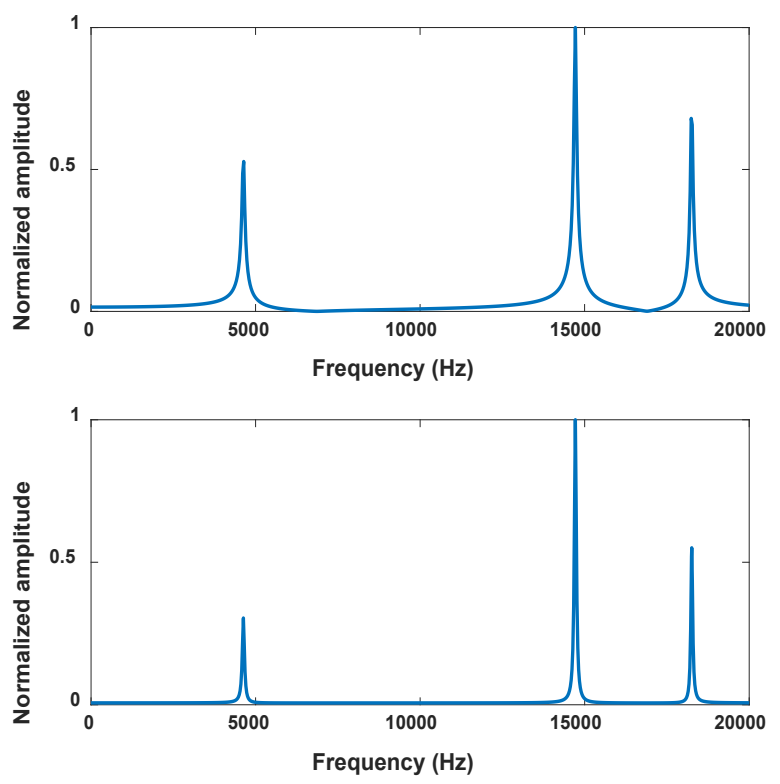

Fig.3. Spectra of impulse response of 3-dof system: spectrum obtained using FFT (at the top); spectrum obtained using in-the-loop computing (at the bottom).

Then, a thousand impulse responses were synthesized by sampling frequency $f_{s}=65536 \mathrm{~Hz}$ as often used in measuring tasks when signals of vibration or sound are acquired and analyzed. The length of IRs was fixed to $N=2048$ samples in length which allowed all possible impulse responses to be completely decaying.

When spectrum is obtained using FFT, the frequency resolution $\Delta f_{F F T}$ is equal to the quotient $f_{s} / N$ [19]. In this case, theoretical frequency error in natural frequency estimation is in the range $\pm \Delta f_{F F T} / 2$. Considering sampling frequency and length of IR mentioned above, frequency resolution using FFT equals $32 \mathrm{~Hz}$ and errors of natural frequency estimation are in the range $\pm 16 \mathrm{~Hz}$. After using FFT in practice, the same range of errors has been obtained as shown in Fig.4. The frequency error was calculated here as the difference between the estimated natural frequency on the base of estimated spectrum and the value already known before the determination of the spectrum as randomized value of damped natural frequency $f_{d r}$ in (4).

Using in-the-loop technique, frequency resolution $\Delta f_{s c r}$ is not related to impulse response length at all. The frequency resolution can be arbitrary given. For the purposes of this numerical study it can be fixed to $0.1 \mathrm{~Hz}$. Frequency errors after comparing known value of natural frequency to frequency estimated using in-the-loop computing are presented in Fig.5. As we can see here, the natural frequency estimation errors are much below the range when using FFT. In practice, further increasing the frequency resolution results in a further reduction in the spread of errors in the estimation of natural frequencies.

The numerical study results also show that the error in frequency estimation using in-the-loop computing does not always have a sign in both directions. It can be either only positive or only negative. 
a)

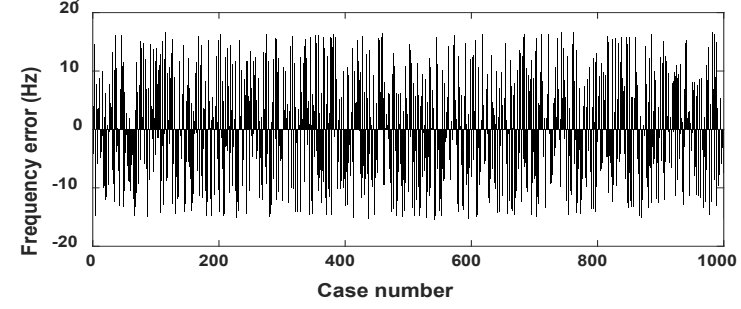

b)

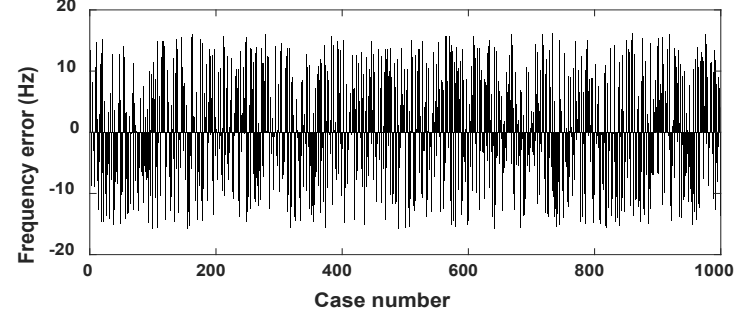

c)

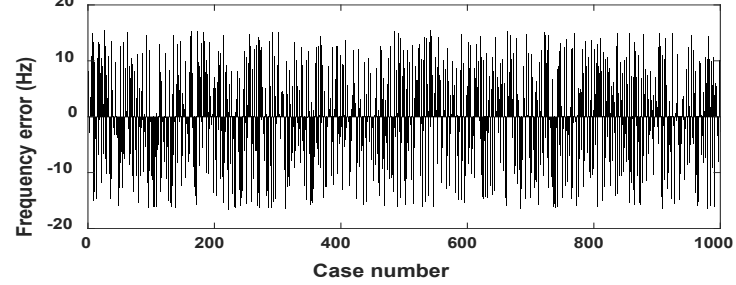

Fig.4. Errors in natural frequency estimation using FFT: a) estimating $f_{d l}$, b) estimating $f_{d 2}, \mathrm{c}$ ) estimating $f_{d 3}$.

a)

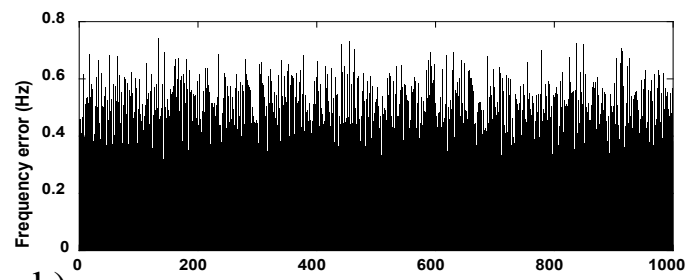

b)

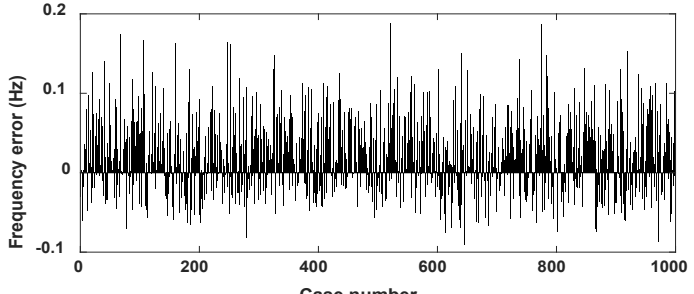

c)

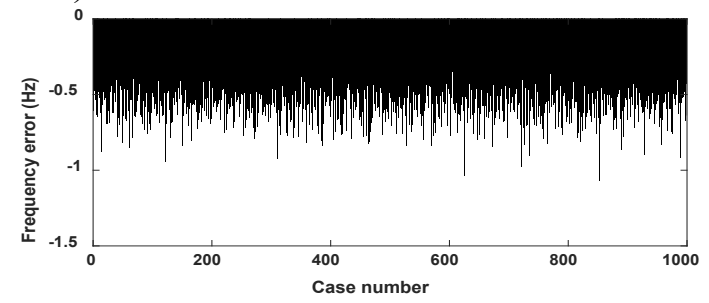

Fig.5. Errors in natural frequency estimation using in-the-loop computing: a) estimating $f_{d 1}$, b) estimating $f_{d 2}$, c) estimating $f_{d 3}$.

\section{RESULTS FOR REAL DATA}

In this testing, data from the measurement of acoustic impulse response of an axial compressor blade were taken into consideration. This impulse response was obtained by manually hitting the blade with an impact hammer in a point placed at the convex side of the blade body. The blade was rigidly mounted in the suitably profiled jaws of a vice. General view of the test stand and experimental setup are shown in Fig.6. and Fig.7., respectively.

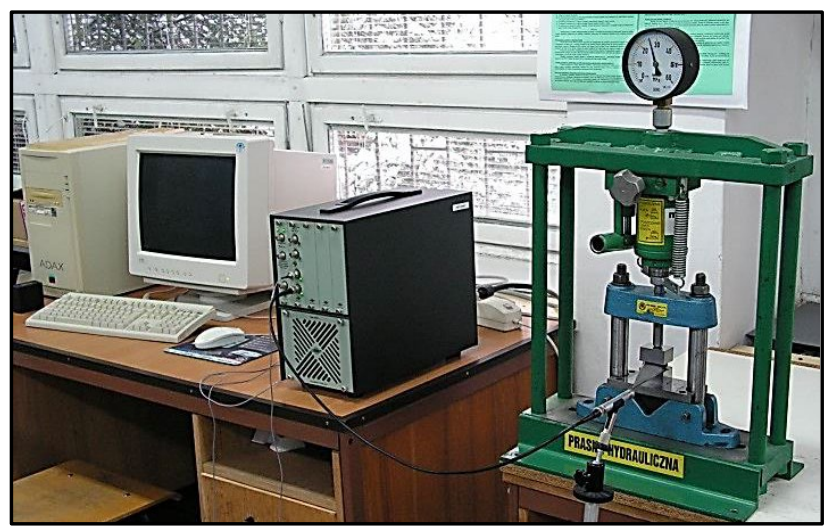

Fig.6. General view of the test stand.

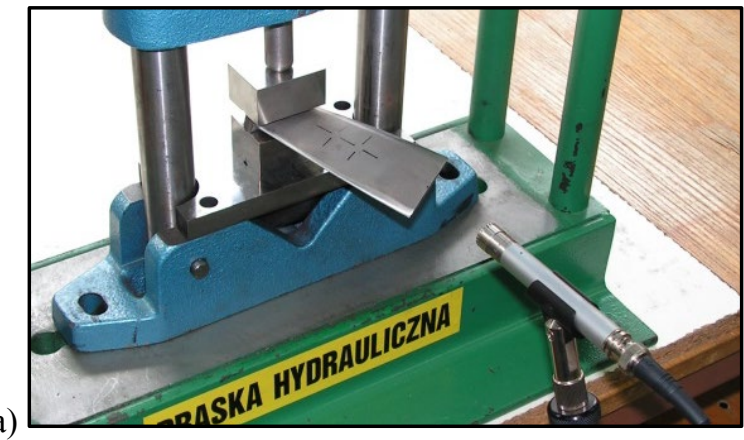

b)

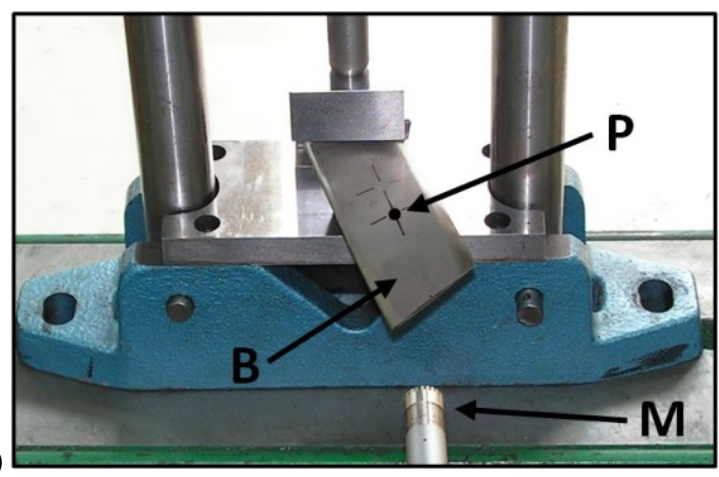

c)

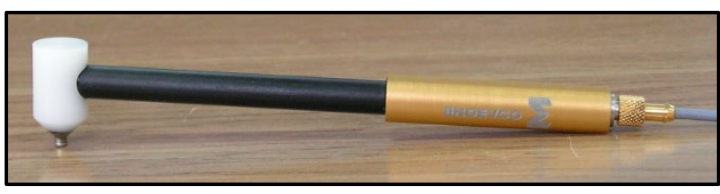

Fig.7. Experimental setup: a) blade mounted using hydraulic press; b) $\mathrm{M}$ - microphone, $\mathrm{B}$ - blade body, $\mathrm{P}$ - point of impulse excitation; c) impact hammer used for impulse excitation. 
The acoustical impulse response was measured with a free field microphone and 2048 samples in length were recorded at the sampling rate of $65536 \mathrm{~Hz}$.

The method verified for really measured IRs has revealed that phase shifting of harmonics makes the spectrum very illegible. This problem has been solved by using the autocorrelation function for IR's time history. The autocorrelation function was obtained in normalized sequence to have at zero lag the value of 1.0. Then, it is natural to choose the cosine function (see Fig.1.) as sweeping operator for having the spectrum amplitudes.

The acoustic impulse response has been recorded by sampling frequency equal to $65536 \mathrm{~Hz}$ and 2048 samples in length. Then, the frequency resolution for spectra resulting from FFT is $32 \mathrm{~Hz}$, whereas spectral frequency resolution in in-the-loop computing can be freely determined, as already mentioned in Section 2. Spectral frequency resolution can be significantly below the resolution of $32 \mathrm{~Hz}$, e.g. $0.1 \mathrm{~Hz}$. Apart from the in-the-loop computing method, FFT with zeropadding technique has been utilized to have reference point to which errors of frequency estimation are calculated.

The acoustic impulse response of the blade is presented in Fig. 8. Spectra obtained both using classical FFT and in-theloop computing are presented in Fig.9.

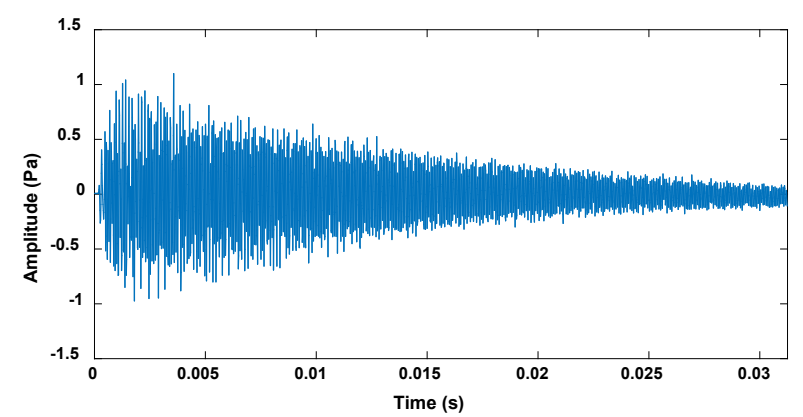

Fig.8. Acoustical impulse response of the blade.

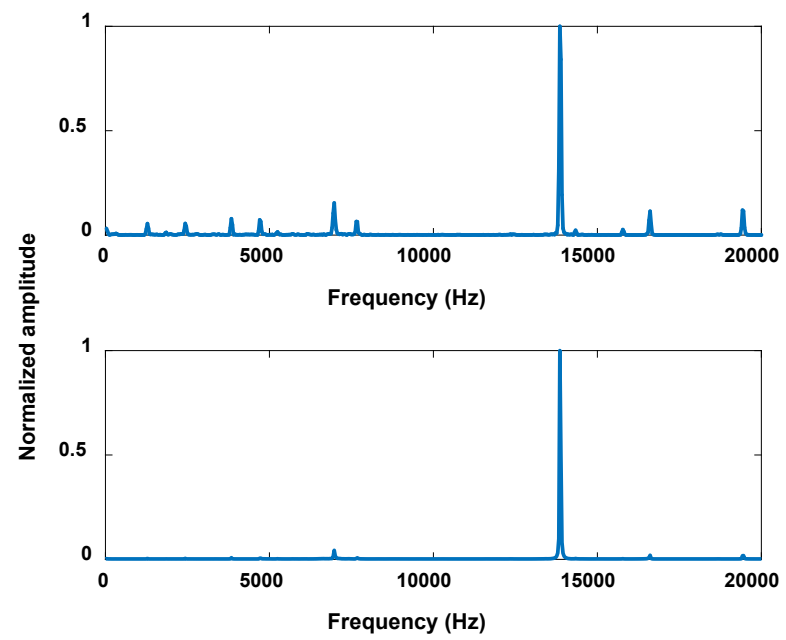

Fig.9. Spectra of acoustic impulse response: obtained using FFT (at the top), obtained using in-the-loop computing (at the bottom).
One dominant harmonic is observed. This is the case when the position of observed harmonic on the frequency scale depends on the type of a tip of the impact hammer and/or point of excitation is in different place of the impulse excitation of object under testing. The results of estimation of natural frequency of the blade are graphically represented in Fig.10. The differences in natural frequency estimation between results obtained using FFT with zero-padding (as reference technique) and in-the-loop method are in the range from 0 to $0.5 \mathrm{~Hz}$.

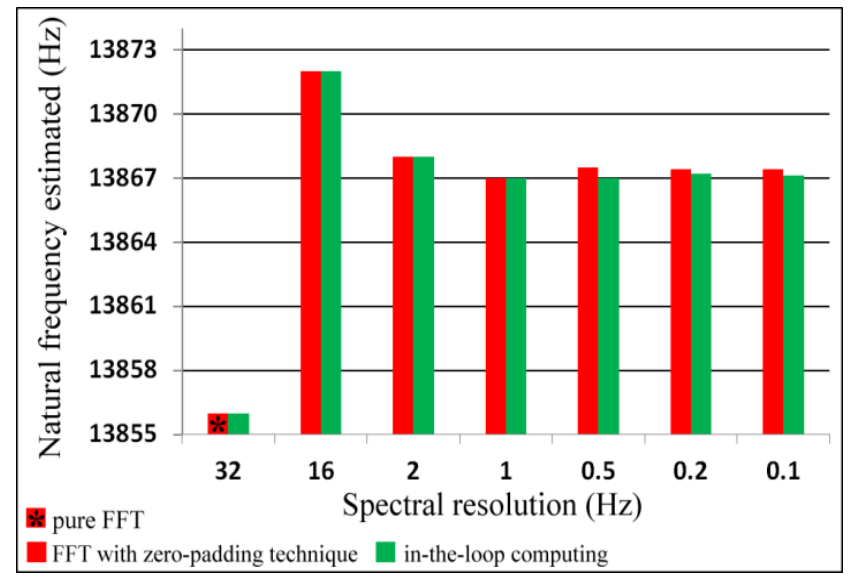

Fig.10. Estimated natural frequency using FFT with zero-padding technique (red) and in-the-loop computing (green).

The determined value of the natural frequency is obviously one of the many natural frequencies of the considered blade. In the given example, the dominant form of blade response is in the frequency range up to several $\mathrm{kHz}$. It is known that other methods of blade excitation (as well as other materials of the tip of the impact hammer) make it possible to observe the values of natural frequencies in many other ranges. However, different approaches to obtain impulse responses, including acoustic ones, do not change the assumptions and basics of the presented method for improving the accuracy of natural frequency measurement.

\section{CONCLUSIONS}

The method presented in the paper creates new possibilities for improving the accuracy of natural frequency measurement when impulse tests are performed and using the classical FFT in signal analysis. These assumptions were tested in a numerical simulation and experimentally on the example of a rotor machine blade. It was made possible by the enhancement of frequency resolution of the impulse response spectrum with in-the-loop computing. It has been proved that the proposed method allows to obtain the spectrum with arbitrarily increased frequency resolution regardless of the length of the analyzed impulse response, which is not allowed by the FFT algorithm. In practice, natural frequencies can be more precisely identified.

The presented method can be very useful in cases where frequency resolution using pure FFT is insufficient due to the accuracy of natural frequency estimation. The method of inthe-loop computing allows then to reduce maximum possible 
error in natural frequency measurements many times in relation to FFT. In addition, the proposed method is based only on the original length of the impulse response without artificial lengthening required when using FFT with zeropadding technique.

\section{ACKNOWLEDGMENT}

This work was supported by Bialystok University of Technology, Faculty of Electrical Engineering, under the work No. WZ/WE-IA/4/2020.

\section{REFERENCES}

[1] Yang, J.-Y., Xia, B.-H., Chen, Z., Li, T.-L., Liu, R. (2020). Vibration-based Structural damage identification: A Review. International Journal of Robotics and Automation, 35 (2), 123-131.

[2] Avci, O., Abdeljaber, O., Kiranyaz, S., Hussein, M. (2021). A review of vibration-based damage detection in civil structures: From traditional methods to Machine Learning and Deep Learning applications. Mechanical Systems and Signal Processing, 147, 1-45.

[3] Glowacz, A., Glowacz, W., Kozik, J., Piech, K., Gutten, M., Caesarendra, W., Liu, H., Brumercik, F., Irfan, M., Khan, Z.F. (2019). Detection of deterioration of threephase induction motor using vibration signals. Measurement Science Review, 19 (6), 241-249.

[4] Ostachowicz, W.M., Krawczuk, M. (1991). Analysis of the effect of cracks on the natural frequencies of a cantilever beam. Journal of Sound and Vibration, 150, 191-201.

[5] Palacz, M., Krawczuk, M. (2002). Vibration parameters for damage detection in structures. Journal of Sound and Vibration, 249, 999-1010.

[6] Dahak, M., Touat, N., Kharoubi, M. (2019). Damage detection in beam through change in measured frequency and undamaged curvature mode shape. Inverse Problems in Science and Engineering, 27 (1), 89-114.

[7] Sha, G., Radzieński, M., Cao, M., Ostachowicz, W. (2019). A novel method for single and multiple damage detection in beams using relative natural frequency changes. Mechanical Systems and Signal Processing, 132, 335-352.

[8] Surace, C., Bovsunovsky, A. (2020). The use of frequency ratios to diagnose structural damage in varying environmental conditions. Mechanical Systems and Signal Processing, 136, 106523.

[9] Salawu, O.S. (1997). Detection of structural damage through changes in frequency: A review. Engineering Structures, 19, 718-723.

[10] Behera, S.K., Parhi, D.R., Das, H.C. (2018). Numerical, experimental and fuzzy logic applications for investigation of crack location and crack depth estimation in a free-free aluminum beam. Vibrations in Physical Systems, 29, 1-20.
[11] Gillich, G.-R., Maia, N.M.M., Mituletu, I.C. (2018). Problem of detecting damage through natural frequency changes. In Vibration-Based Techniques for Damage Detection and Localization in Engineering Structures. World Scientific, vol. 10, 105-140.

[12] Gillich, G.-R., Mituletu, I.C. (2017). Signal postprocessing for accurate evaluation of the natural frequencies. In Structural Health Monitoring: An Advanced Signal Processing Perspective, Springer, vol. 26, 13-37.

[13] Luczynski, M., Dobrucki, A., Brachmanski, S. (2020). Active tone elimination algorithm using FFT with interpolation and zero-padding. In Signal Processing: Algorithms, Architectures, Arrangements, and Applications (SPA 2020) Conference Proceedings, SPA. IEEE, 163-168.

[14] Quinn, B.G. (1994). Estimating frequency by interpolation using Fourier coefficients. IEEE Transactions on Signal Processing, 42 (5), 1264-1268.

[15] Luo, J., Xie, Z., Xie, M. (2016). Interpolated DFT algorithms with zero padding for classic windows. Mechanical Systems and Signal Processing, 70-71, 1011-1025.

[16] Shen, T.-A., Li, H.-N., Zhang, Q.-X., Li, M. (2017). A novel adaptive frequency estimation algorithm based on interpolation FFT and improved adaptive notch filter. Measurement Science Review, 17, 48-52.

[17] Mituletu, I.C., Gillich, G.-R., Maia, N.M.M. (2019). A method for an accurate estimation of natural frequencies using swept-sine acoustic excitation. Mechanical Systems and Signal Processing, 116, 693-709.

[18] Barot, T., Burgsteiner, H., Kolleritsch, W. (2020). Comparison of discrete autocorrelation functions with regards to statistical significance. In Applied Informatics and Cybernetics in Intelligent Systems: Proceedings of the 9th Computer Science On-line Conference 2020. Springer, vol. 3, 257-266.

[19] Kolokolov, A.S., Lyubinskii, I.A. (2019). Measuring the pitch of a speech signal using the autocorrelation function. Automation and Remote Control, 80, 317-323.

[20] Ahn, S.J., Jeong, W.B., Yoo, W.S. (2005). Improvement of impulse response spectrum and its application. Journal of Sound and Vibration, 288, 12231239. 